\title{
Erratum: 2-Hydroxy-3-methoxybenzoic acid attenuates mast cell-mediated allergic reaction in mice via modulation of the FceRI signaling pathway
}

Kim YY, Je IG, Kim MJ, Kang BC, Choi YA, Baek MC, et al.

Acta Pharmacol Sin 2017; 38: 90-99. doi: 10.1038/aps.2016.112.

The affiliation in PubMed is wrong.

The correct affiliation is

${ }^{1}$ CMRI, Department of Pharmacology, Daegu 700-422, Republic of Korea; ${ }^{2}$ Research Laboratories, ILDONG Pharmaceutical Co Ltd, Hwaseong 18449, Republic of Korea; ${ }^{3}$ Department of Molecular Medicine, Daegu 700-422, Republic of Korea; ${ }^{4}$ Department of Biochemistry and Cell Biology, School of Medicine, Kyungpook National University, Daegu 700-422, Republic of Korea; ${ }^{5}$ Molecular Immunology Section, Laboratory of Immunology, National Eye Institute, National Institutes of Health, Bethesda, MD 20892, USA; ${ }^{6}$ College of Pharmacy, Woosuk University, Jeonju 565-701, Republic of Korea; ${ }^{7}$ Natural Product Research Center, Korea Research Institute of Bioscience and Biotechnology, Jeongeup 580-185, Republic of Korea; ${ }^{8}$ National Primate Research Center, Korea Research Institute of Bioscience and Biotechnology, Ochang 28116, Republic of Korea; ${ }^{9}$ Department of Molecular Medicine, School of Medicine, Gachon University, Incheon 406-840, Republic of Korea 